\title{
Linc02527 promoted autophagy in Intrahepatic cholestasis of pregnancy
}

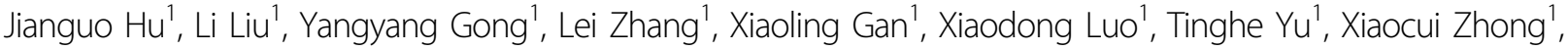 \\ Xinru Deng ${ }^{1}$, Lina $\mathrm{Hu}^{1}$, Zhanyu Zhang ${ }^{1}$ and Xiaojing Dong ${ }^{1}$
}

\begin{abstract}
LncRNA plays a crucial role in human disease. However, the expression and function of LncRNA in ICP(Intrahepatic cholestasis of pregnancy) is still not fully elucidated. In this study, we found Linc02527 was increased expression in placenta and serum of ICP patients. Ectopically expression of Linc02527 promoted autophagy and proliferate in HTR8 cells. Silencing Linc02527 suppressed the autophagy and proliferate in HTR8 cells. Mechanically study revealed that Linc02527 regulated the expression of ATG5 and ATG7 by sponging miR-3185. Linc02527 directly binding to YBX1 and activated P21. The growth of C57 mouse was retarded when autophagy was activated. In normal condition, inhibited autophagy using chloroquine did not affect the growth of C57 mouse. However, in the condition of autophagy was activated, inhibited autophagy using chloroquine can improve the growth of C57 mouse. Overall, the results of this study identified Linc02527 as a candidate biomarker in ICP and a potential target for ICP therapy. Chloroquine was a potential drug for ICP therapy.
\end{abstract}

\section{Introduction}

Intrahepatic cholestasis of pregnancy (ICP) is an obstetrical complication. It may lead to intrauterine fetal death. The hazard behind ICP is the excessive accumulation of bile acids in hepatocytes and the inhibition of bile excretion ${ }^{1}$. Overproduction of bile acids or their impaired removal from hepatocytes may be the reason of this pathology, which leads to increased bile acid serum concentration ${ }^{1-4}$.

Autophagy is an evolutionarily conserved process by which cytosolic components are sequestered into doublemembrane vesicles and consequently fused with lysosomes ${ }^{5,6}$. Autophagy plays an important role in cellular pathways, including in growth, development and differentiation, aging and cell death ${ }^{7-9}$. Previous study reported that autophagy was involved in the progress of preeclampsia $^{10}$. The marker of autophagy was abnormally expression in placenta with hypertensive disorders in

\footnotetext{
Correspondence: Xiaojing Dong (xffdoctor@163.com)

'Department of Obstetrics and Gynecology, Second Affiliated Hospital,

Chongqing Medical University, Chongqing, China

These authors contributed equally: Jianguo Hu, Li Liu, Yangyang Gong.

Edited by G.M. Fimia
}

pregnancy ${ }^{11}$. However, little is known about the function of autophagy in ICP.

LncRNA has been demonstrated that played an important role in human disease. LncRNA HOTAIR ${ }^{12}$, lncRNA MACC1-AS1 ${ }^{13}$, LncRNA-MALAT1 ${ }^{14}$ and LncRNA NEAT $1{ }^{15}$ were involved in cancer migration and invasion. In human placenta, some LncRNAs was vrefiied. Misregulation of LOC391533, LOC284100 and CEACAMP8 may contribute to the mechanism underlying preeclampsia $^{16}$. IncRHOXF1 is the first IncRNA that regulates the host response to viral infections in human placental progenitor cells ${ }^{17}$. However, little is known about the expression and function of LncRNA in ICP.

In the present study, we investigated LncRNA expression and its potential clinical significance in ICP, trying to clarify the possible function of LncRNA in the pathogenesis.

\section{Materials and methods \\ Tissue specimens}

The placenta tissue and serum sample was were obtained from the second affiliated Department of

\section{(c) The Author(s) 2018}

(c) (i) Open Access This article is licensed under a Creative Commons Attribution 4.0 International License, which permits use, sharing, adaptation, distribution and reproduction c. in any medium or format, as long as you give appropriate credit to the original author(s) and the source, provide a link to the Creative Commons license, and indicate if changes were made. The images or other third party material in this article are included in the article's Creative Commons license, unless indicated otherwise in a credit line to the material. If material is not included in the article's Creative Commons license and your intended use is not permitted by statutory regulation or exceeds the permitted use, you will need to obtain permission directly from the copyright holder. To view a copy of this license, visit http://creativecommons.org/licenses/by/4.0/. 
Obstetrics and Gynecology, Second Affiliated Hospital, Chongqing Medical University. The Ethics Committee of the Chongqing Medical University approved the study documents and the use of archived placenta tissues and serum sample.

\section{Animal models}

Thirty Sprague-Dawley pregnant rats were randomly divided into two groups: Group NP and Group ICP. The rats provided by animal experiment center of Chongqing medical university. The rats were maintained in collective cages in an appropriate room with controlled temperature and with a 12-h light cycle and fed with standard rat chow and water. They were aged at $\sim 150-180$ days and weighed $\sim 250-270$ g. From day 13 of gestation, rats in group ICP were injected intraperitoneally with estradiol benzoate ( $5 \mathrm{mg} / \mathrm{kg}$, Abcam) for 7 days consecutively. Group NP (control group, named Group NP) was injected intraperitoneally with normal saline. On day 13 of gestation, the levels of TBA, alanine aminotransferase (ALT), aspartate aminotransferase (AST), and total bile acid(TBA) was detected in serum which was drawn from tail vein of pregnant rats. On day 21 of gestation, cesarean section was performed with anesthesia by intraperitoneal injection of $10 \%$ hydral with dosage of $3 \mathrm{~mL} / \mathrm{kg}$. The placenta was obtained for detecting the expression of LC3-II.

Forty C57 pregnant mice were randomly divided into four groups: group $\mathrm{NC}$, group RAPA, group $\mathrm{CQ}$ and group $\mathrm{CQ}+\mathrm{RAPA}$. Mice were maintained in collective cages in an appropriate room with controlled temperature with a 12-h light cycle, and mice were allowed access to food and water ad libitum. From day 12 of gestation of gestation, mice in group $\mathrm{NC}$ were injected intraperitoneally with normal saline for 6 days consecutively. Group RAPA were injected intraperitoneally with rapamycin $(5 \mathrm{mg} / \mathrm{kg}$, CST). Group CQ were injected intraperitoneally with chloroquine $(50 \mathrm{mg} / \mathrm{kg}$, CST). Group RAPA + CQ were injected intraperitoneally with rapamycin $(5 \mathrm{mg} / \mathrm{kg}, \mathrm{CST})$ and chloroquine $(50 \mathrm{mg} / \mathrm{kg}$, CST). On day 21 of gestation, the pregnant mice were sacrificed. Then, the fetal mice was calculated for body weight, fetus number, bodylenth and placenta weight.

\section{Microarray assay}

Human LncRNA Microarray V4.0 (Agilent, USA) is designed for global profiling of human lncRNAs and protein-coding transcripts. Human miRNA Microarray Release 21.0 (Agilent technologies, USA) is designed for detecting the miRNA expression. The experiments and data analysis were performed by Beijing Boao Crystal Code Biotechnology Co. Ltd.

\section{Cell culture, transfection procedure, and reagents}

Human HTR8 cells were cultured in RPMI 1640 medium (Sigma-Aldrich, R8758) containing 10\% fetal bovineserum. The cells were incubated under $5 \%$ carbon dioxide at $37^{\circ} \mathrm{C}$. siRNA corresponding to the target sequences were synthesized by Genepharma (Shanghai, China). The following sequences were targeted for human Linc02527 small interfering RNA (siRNA): Linc02527-1: 5'-GGAGAGGCUAUAAACUUCUTT-3'; Linc02527-2: 5'-CCGUGCAUAUUGUCUCCAATT-3'; and NC (negative control) siRNA: 5'-UUCUUCGAAGGUGUCACGU TT-3'. Lentiviral vectors expressing shRNA targeting Linc02527 (named LV3-Inc02527-1 and LV3-Inc02527-2) and the Linc02527-lentiviral expression vector (named LV5-Inc02527) were provided by Genepharma. miR-3185 mimics (sense: 5'-AGAAGAAGGCGGUCGGUCUGCG G-3') were synthesized at Ruibo Biotechnology(Guangzhou, China). AST detecting using AST Activity Assay Kit (MAK055, Sigma), ALT detecting using ALT Activity Assay (MAK052, Sigma); TBA detecting using Total Bile Acids (TBA) Assay Kit (Colorimetric) (BioVision.Inc, America); CG detecting using CG RIA Kit(YB-10016, Shanghai Zibo Biotechnology Co., Ltd. China).

\section{Flow cytometric analysis of cell apoptosis}

Cells for apoptotic analysis were double stained with Annexin V-FITC and propidium iodide $48 \mathrm{~h}$ after transfection and analyzed using a flow cytometer (FACScan; BD Biosciences, Shanghai, China) equipped with CellQuest software (BD Biosciences). Each experiment was performed in triplicate.

\section{RNA extraction and Quantitative real-time polymerase chain reaction( $\mathrm{RT}$-qPCR)}

Total RNA was isolated using a pure high-purity Total RNA Rapid Extraction Kit (Bioteke Corporation, RP1201) according to the manufacturer's instruction. cDNA was synthesized using the iSCRIPT cDNA synthesis kit (Bio-Rad Laboratories, 4106228). All of the primers used to amplify lncRNAs were synthesized by GenePharma. The real-time PCR kit was purchased from GeneCopoeia. PCR conditions were $95^{\circ} \mathrm{C}$ for $10 \mathrm{~s}, 60^{\circ} \mathrm{C}$ for $20 \mathrm{~s}$ and $72{ }^{\circ} \mathrm{C}$ for $10 \mathrm{~s}$. Each sample was analyzed in triplicate. Relative quantification of mRNA was performed using the comparative threshold cycle (CT) method. This value was used to plot the gene expression using the formula $2^{-\Delta \Delta \mathrm{CT}}$.

\section{Dual-luciferase reporter gene assay}

Luciferase reporter gene assay was performed using the Dual-Luciferase Reporter Assay System (Promega Corporation, E1910) according to the manufacturer's instruction. Luciferase reporter assays for ATG5, ATG7 and Linc02527, wild-type or mutant reporter constructs (termed WT or Mut; obtained from Genepharma) were cotransfected into HTR8 cells in 24-well plates with $100 \mathrm{nM}$ MIR3185 or $100 \mathrm{nM}$ miR-NC and Renilla plasmid 
Table 1 Summary of IncRNA that are differently expressed between placenta from NP and ICP (ICP vs. NP)

\begin{tabular}{llll}
\hline Gene symbol & Chromosomal location & Regulation & P-value \\
\hline LINC02027 & Chr3:810430188-81144798(+) & Up & 0.02699606 \\
LINC02527 & Chr6:112221691-112223054(-) & Up & 0.01485026 \\
FAM239A & ChrX: $3771050-3781615(-)$ & Up & 0.025825039 \\
KCNMB2-AS1 & Chr3:178447071-178577338(-) & 0.028023811 \\
YEATS2-AS1 & Chr3:183524244-183526729(-) & Up & 0.0014545523 \\
LINC02050 & Chr3: 80814047-80838505(+) & Up & 0.09413093 \\
TCONS_00005551 & Chr3:81143793-81144462(+) & 0.02998128 \\
TCONS_00011228 & Chr6:112221561-112230600(-) & 0.014197185 \\
LINC01436 & Chr21: $36005338-36007838(+)$ & Up & 0.010051289 \\
HIT000075265 & Chr16:23315293-23360088(+) & Up & 0.021368366 \\
uC021wnb.1 & Chr22: $24245625-24247864(-)$ & Up & 0.024611032 \\
\hline
\end{tabular}

by using Endofectin-Plus (Gene-Copoeia, Z01010A). All experiments were performed at least 3 times.

\section{Western blotting}

The expressions of LC3, ATG7, ATG5, YBX1, P21 and actin proteins were analyzed by western blot ${ }^{18,19}$. The primary antibodies used include polyclonal rabbit antiLC3 (Cell Signaling Technology, 4108); rabbit monoclonal to ATG5 (Abcam, ab109490); rabbit monoclonal to ATG7 (Abcam, ab183188); rabbit monoclonal to YBX1 (Abcam, ab76149); rabbit monoclonal to P21 (Abcam, ab109520); and monoclonal rabbit anti-beta actin (Abcam, ab115777). The band density was analyzed using a gel imaging system and compared with an internal control.

\section{Cell proliferation assay}

Cell proliferation was determined using the EdU assay. It was performed using the Cell-Light TM EdU imaging detecting kit according to the instructions in the kit (Ruibo Biotechnology, Guangzhou, China) ${ }^{20,21}$.

RNA pulldown assay and matrix-assisted laser desorption/ ionization time of flight mass spectrometry (MALDI-TOFMS)

To find the proteins bonding to Linc02527 directly, the RNA pull-down assay was performed ${ }^{22,23}$. The specific operation steps was according to the previous study ${ }^{24}$. Using SDS-PAGE gel electrophoresis, Silver stain and MALDI-TOF-MS, then, the specific proteins were identified.

\section{Statistical analysis}

All statistical analyses were performed using SPSS software, version 17.0 (Chicago, IL). Each experiment was performed in triplicate. Statistical analysis was performed by Student t test. Pearson correlation analysis was conducted to analyze the association between Linc02527 expression and serum TBA, CG, ALT or AST. Data were presented as mean \pm standard deviation. Statistical significance was defined as a p-value less than 0.05 .

\section{Results}

Microarray expression profiles of IncRNAs and mRNAs in placenta of ICP patients

To explore the potential biological functions of lncRNAs in ICP, we examined the expression patterns of lncRNAs and mRNAs in placenta of ICP patients and controls with full term pregnancy. In total, 358 lncRNAs and 255 mRNAs showed a $\geq 1.5$-fold change $(P<0.05)$ in 8 samples (Fig. 1a, b). Of these, 100 lncRNAs were upregulated, and 258 were down-regulated; 123 mRNAs were up-regulated, 132 were down-regulated (Table 1 and S1). The differential expression lncRNAs were related to respiratory diseases, hypertensive diseases, cardiovascular diseases and so on (Fig. 1c). These lncRNAs were involved in many cellular process including in sodium channel activity, peptide disulfide oxdoreductase activity and so on (Fig. 1d). They also participated in aldosterone-regulated sodium reabsorption, Stimuli-sensing channels, glutathine metabolism and transmembrane transport of small molecules (Fig. 1e).

\section{Linc02527 expression was increased in placenta and serum of ICP}

We verified the expression of 11 LncRNA in placenta of ICP. We found that Linc02527, KCNMB2-AS1, YEATS2AS1 and TCONS-00011228 were significant increased in placenta of ICP (Fig. 2a, b, Fig. 2S1). We also observed Linc02527, KCNMB2-AS1, YEATS2-AS1 and TCONS00011228 expression was significantly increased in serum 


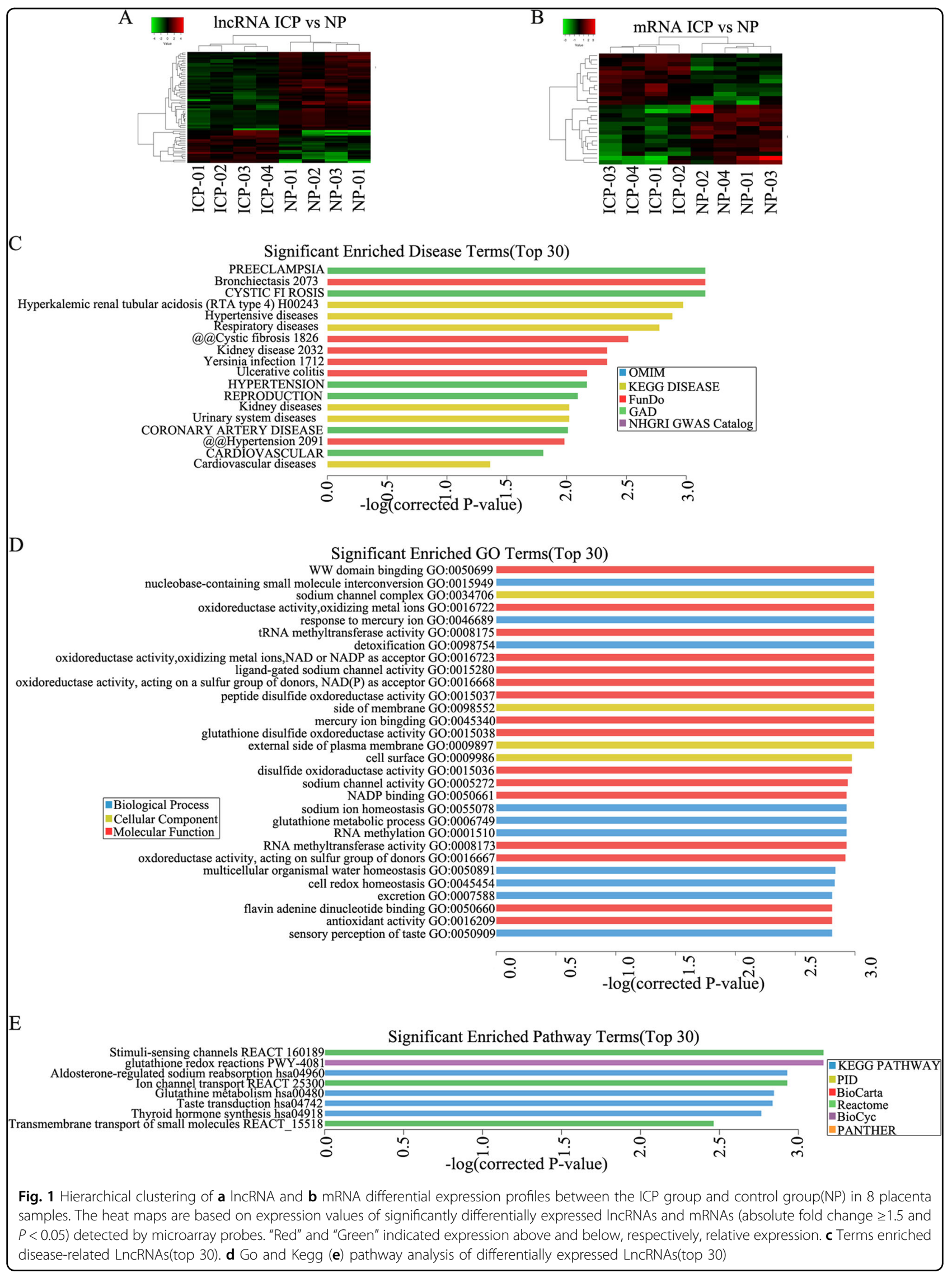




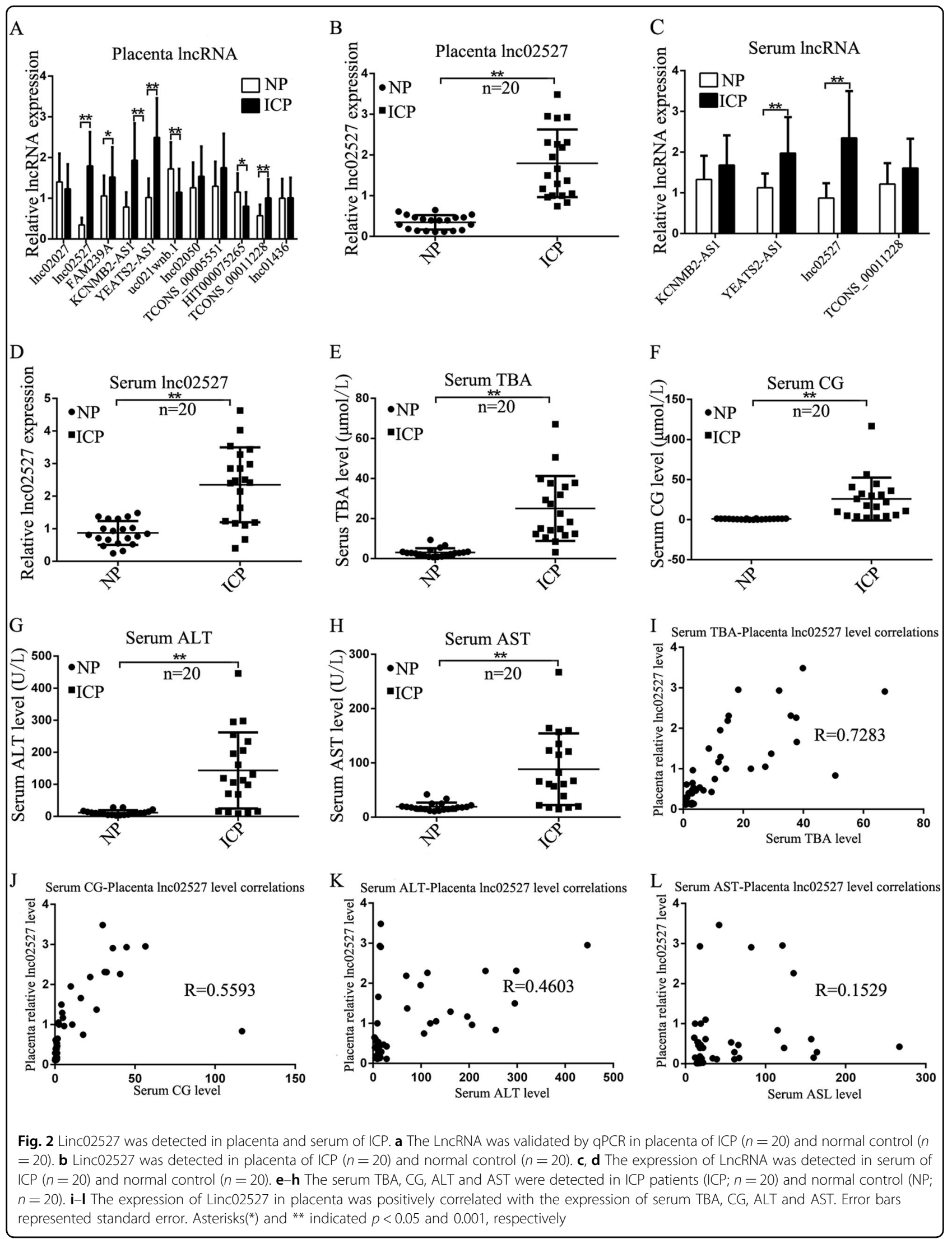


Table 2 Biochemistry (mean \pm SD)

\begin{tabular}{llll}
\hline Parameter & NP $(\boldsymbol{n}=\mathbf{2 0})$ & ICP $(\boldsymbol{n}=\mathbf{2 0})$ & $\boldsymbol{P}$ value \\
\hline TBA $(\mu \mathrm{mol} / \mathrm{L})$ & $3.06 \pm 2.12$ & $25.04 \pm 16.19$ & $<0.01$ \\
CG $(\mu \mathrm{mol} / \mathrm{L})$ & $0.85 \pm 0.49$ & $25.71 \pm 26.66$ & $<0.01$ \\
ALT $(\mathrm{U} / \mathrm{L})$ & $11.75 \pm 7.30$ & $143.25 \pm 118.48$ & $<0.01$ \\
AST $(\mathrm{U} / \mathrm{L})$ & $19.35 \pm 7.55$ & $88.4 \pm 66.05$ & $<0.01$ \\
Total bilirubin $(\mu \mathrm{mol} / \mathrm{L})$ & $9.10 \pm 2.62$ & $11.64 \pm 5.39$ & $\mathrm{NS}$ \\
Direct bilirubin $(\mu \mathrm{mol} / \mathrm{L})$ & $3.11 \pm 1.49$ & $4.92 \pm 3.64$ & $<0.05$ \\
Indirect bilirubin $(\mu \mathrm{mol} / \mathrm{L})$ & $6.00 \pm 2.19$ & $6.72 \pm 3.15$ & $\mathrm{NS}$ \\
\hline
\end{tabular}

of ICP (Fig. 2c, d and Fig. S2A-C). The serum TBA, CG, ALT and AST was increased in ICP compared with that normal pregnant (Fig. 2e-h and Table 2). The Birth body length of newborn in ICP group was shorter than that of normal group (Table 3). The serum TBA, CG, ALT and AST was positively correlated with the expression of Linc02527 in placenta (Fig. 2i-l). The serum TBA, CG, ALT and AST was positively correlated with the expression of Linc02527 in serum (Fig. 2 S2D-G).

\section{Linc02527 promoted proliferation and autophagy in HTR8 cells}

We next determined the function of Linc02527. We firstly detected the apoptosis rate after silencing or ectopically expressed Linc02527 in HTR8 cells. The apoptosis rate is not significant difference between HTR8 cells infected with LV3-lnc02527-1 or lnc02527-2 and HTR8 cells infected with LV3-NC (Fig. 3a). Also, the apoptosis rate is not changed in HTR8 cells infected with LV5lnc02527 compared with infected with LV5-NC (Fig. 3c). The expression of BCL-2 and Bax was not changed when silencing or ectopically expressed Linc02527 in HTR8 cells (Fig. 3b, d). This data revealed that Linc02527 did not regulate apoptosis in HTR8 cells. So we detected whether Linc02527 regulated autophagy in HTR8 cells. As we expected, the expression of LC3-II was decreased in HTR8 cells infected LV3-lnc02527-1 or lnc02527-2 compared to that infected LV3-NC (Fig. 3b). The expression of LC3-II was increased in HTR8 cells infected LV5-Inc02527 compared to that infected LV5-NC (Fig. 3d).

To investigate whether Linc02527 regulated autophagic flux, we quantified the levels of LC3-II, the autophagosome associated form of the protein, in the absence and presence of the lysosomal inhibitor chloroquine. The ratio between the LC3-II levels with and without chloroquine as an index of overall autophagic flux ${ }^{25}$. We observed a increase of this ratio in HTR8 cells infected LV5-lnc02527 compared to that infected LV5-NC (Fig. 3e).This data indicated that Linc02527 promoted autophagic flux. We
Table 3 Subject demographic and characteristics (mean \pm SD)

\begin{tabular}{llll}
\hline Parameter & NP $(\boldsymbol{n}=\mathbf{2 0})$ & ICP $(\boldsymbol{n}=\mathbf{2 0})$ & $\boldsymbol{P}$ value \\
\hline Age (years) & $30.95 \pm 3.35$ & $29.20 \pm 4.34$ & NS \\
Height (cm) & $160.45 \pm 4.84$ & $159.70 \pm 2.68$ & NS \\
Weight (kg) & $70.325 \pm 7.87$ & $67.25 \pm 8.19$ & NS \\
Weight gain (kg) & $14.275 \pm 4.31$ & $13.625 \pm 2.86$ & NS \\
Gravidity & $2.30 \pm 1.78$ & $2.45 \pm 1.76$ & NS \\
Parity & $1.25 \pm 0.44$ & $1.3 \pm 0.47$ & NS \\
Gestational weeks at delivery & $38.39 \pm 0.95$ & $37.82 \pm 1.03$ & NS \\
Birthweight (kg) & $3.2 \pm 0.32$ & $3.04 \pm 0.44$ & NS \\
Birth body length (cm) & $50.35 \pm 1.27$ & $48.85 \pm 1.90$ & $<0.01$ \\
\hline
\end{tabular}

determined whether autophagy was activated in the placenta of ICP. We found that the expression of LC3-II, ATG5, ATG7 and Beclin1 was increased in placenta of ICP compared to normal pregnant (Fig. 3f).

We also investigated whether Linc02527 was involved in the proliferate ability. We observed that silencing Linc02527 suppressed cellular proliferate; however, ectopically expressed Linc 02527 promoted the cellular proliferate ability in HTR8 cells (Fig. 3g-i). To determine the mechanism of Linc02527, the location of Linc02527 was detected. Our results revealed that Linc02527 was located in the cytoplasm and nucleus (Fig. 3k).

\section{Linc02527 promoted autophagy by sponging miR-3185}

The expression of Linc02527 was increased in HTR8 cells infected LV5-Inc02527 compared to that infected LV5-NC (Fig. 4a). The differential miRNA expression was screened using miRNA array (S2). We verified six most significant down-regulated genes using qPCR. Six miRNAs (miR-1323, miR-3185, miR-3935, miR-3187, miR3663 and miR-515) was decreased in HTR8 cells infected LV5-Inc02527 compared to that infected LV5-NC (Fig. 4b and S2). Bioinformatics prediction (LncBase Predicted v.2) that there was a binding site between miR-3185 and Linc02527 (Fig. 4c). Luciferase reporter gene assay confirmed the binding of miR-3185 with Linc02527 (Fig. 4d). Over-expression of miR-3185 suppressed the expression of Linc02527 (Fig. 4e). However, silencing miR-3185 increased the expression of Linc02527(Fig. 4f).

Bioinformatics prediction(microRNA.org) that ATG5 and ATG7 were the potential target of miR-3185. So we detected the expression of ATG5 and ATG7 through silencing or over-expression of miR-3185 in HTR8 cells. The expression of ATG5 and ATG7 was decreased after over-expression of miR-3185. In contrast, the expression of ATG5 and ATG7 was increased after silencing miR-3185 (Fig. 5a-c). The LC3-II also was regulated by 


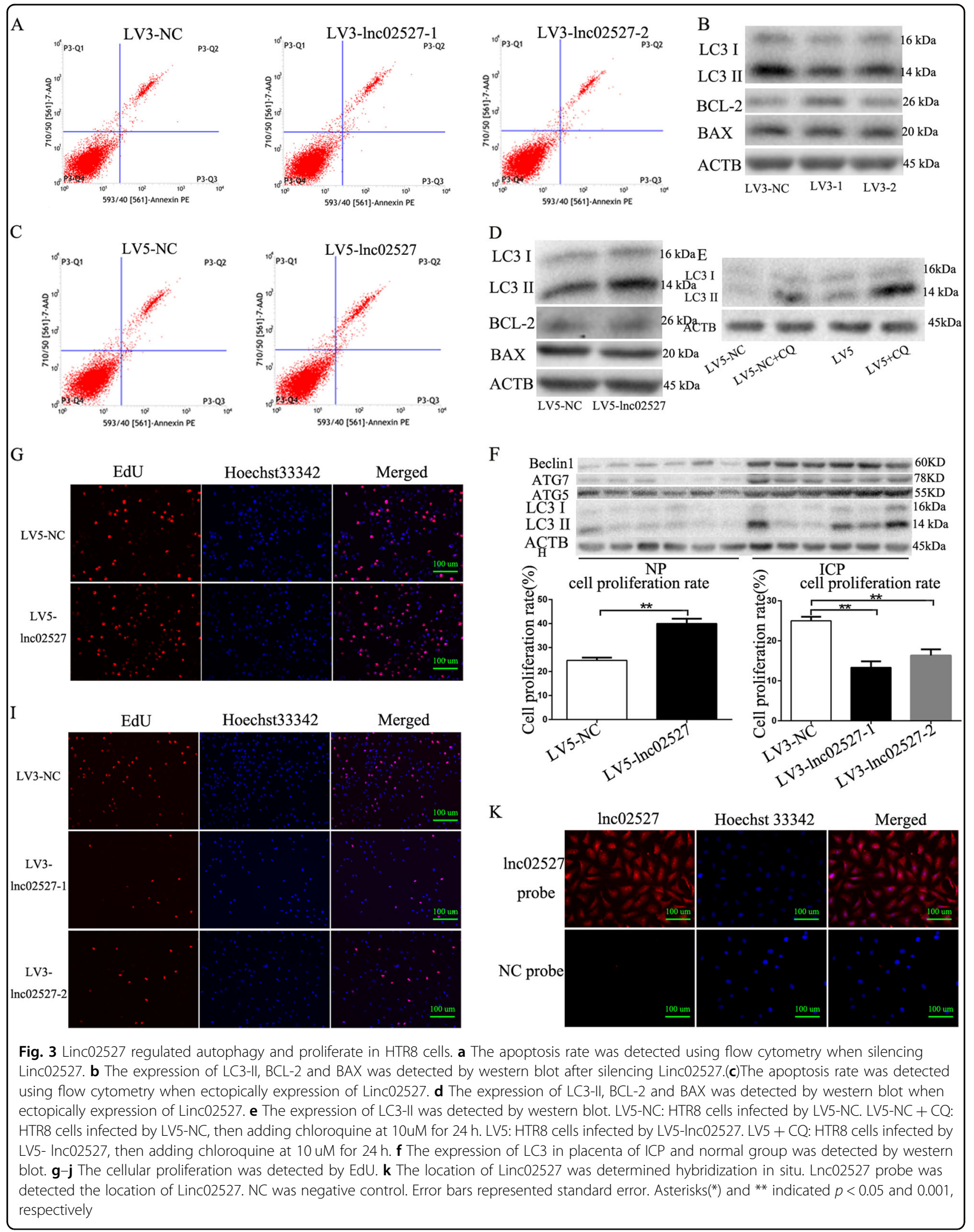




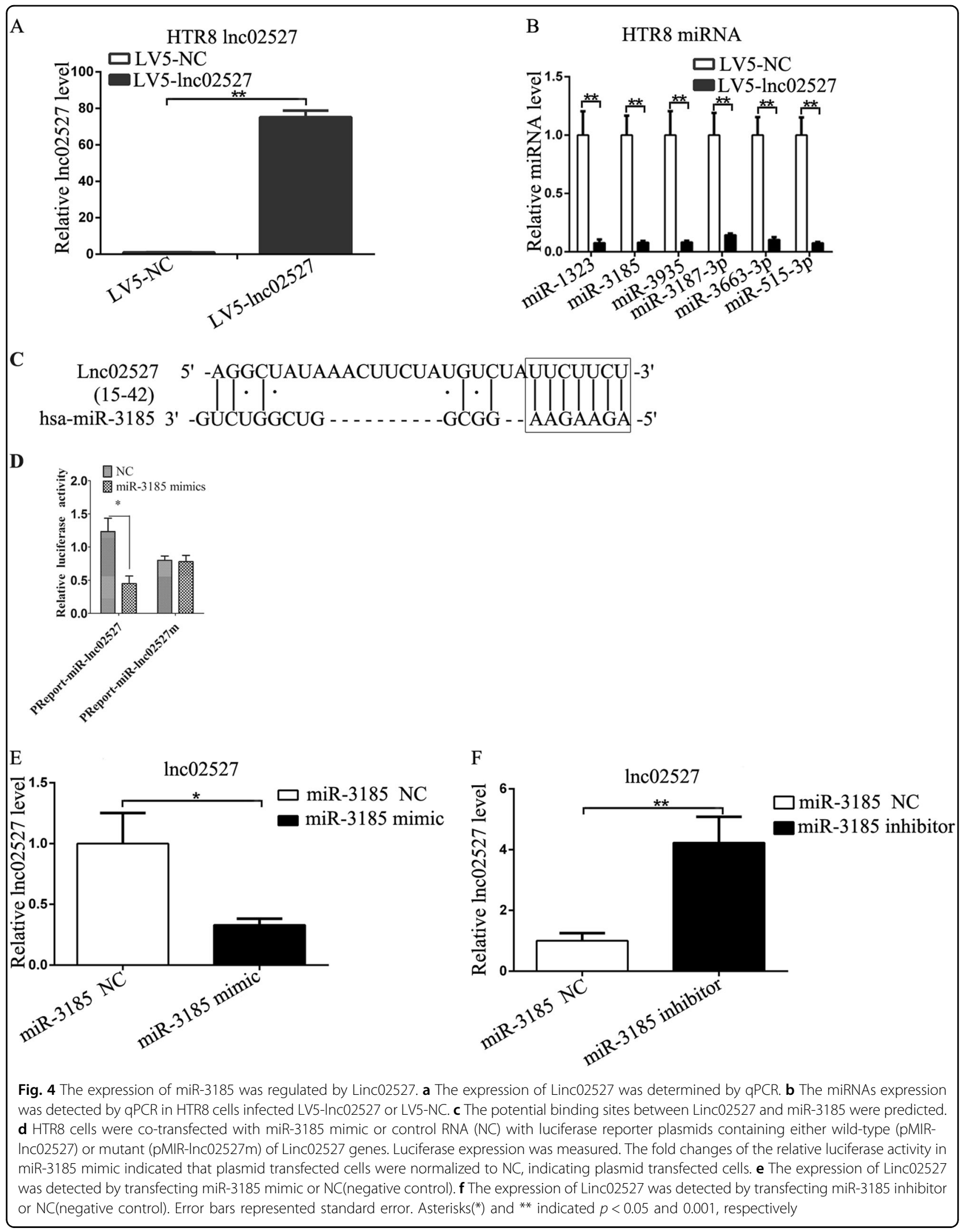




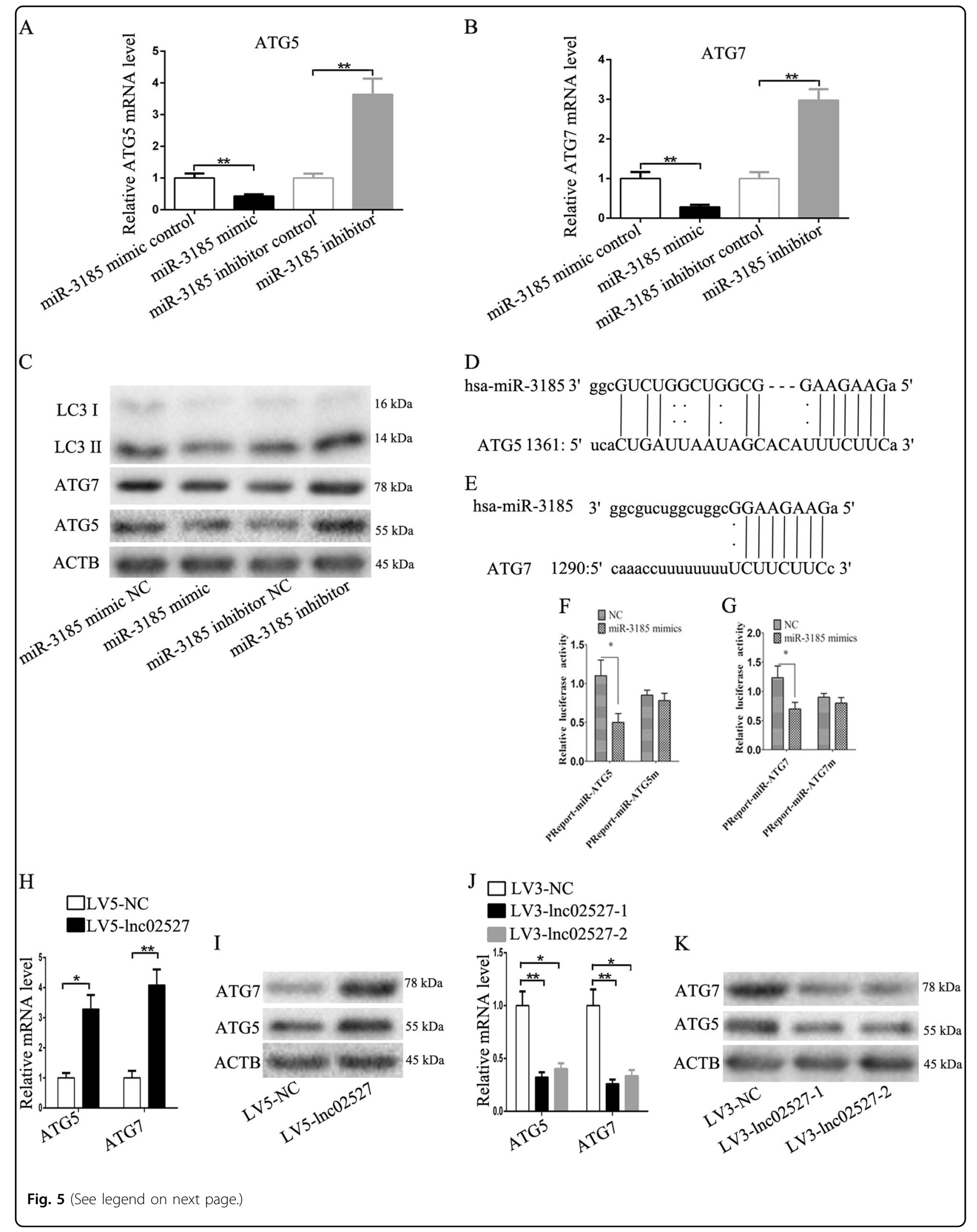


(see figure on previous page)

Fig. 5 The expression of ATG5 and ATG7 was regulated by miR-3185. a The expression of ATG5 mRNA was detected when transfecting with miR3185 mimic, miR-3185 mimic control, miR-3185 inhibitor or miR-3185 inhibitor mimic. b The expression of ATG7 mRNA was detected when transfecting with miR-3185 mimic, miR-3185 mimic control, miR-3185 inhibitor or miR-3185 inhibitor mimic. c The protein expression of ATG5, ATG7 and LC3-II was determined when transfecting with miR-3185 mimic, miR-3185 mimic control, miR-3185 inhibitor or miR-3185 inhibitor mimic. d, e The potential binding sites between miR-3185, ATG5 and ATG7 were predicted. f, $\mathbf{g}$ HTR8 cells were co-transfected with miR-3185 mimic or control RNA (NC) with luciferase reporter plasmids containing either wild-type (pMIR-ATG5-3'UTR and pMIR-ATG7-3'UTR) or mutant (pMIR-ATG53'UTRm and pMIR-ATG7-3'UTRm) of ATG5 and ATG7 genes. Luciferase expression was measured. The fold changes of the relative luciferase activity in miR-3185 mimic indicated that plasmid transfected cells were normalized to NC, indicating plasmid transfected cells. $\mathbf{h}$, i The expression of ATG5 and ATG7 was determined in HTR8 cells infected with LV5-NC or LV5-Inc02527. $\mathbf{j}, \mathbf{k}$ The expression of ATG5 and ATG7 was determined in HTR8 cells infected with LV3-NC, LV2-Inc02527-1 or LV2-Inc02527-2. Error bars represented standard error. Asterisks( $\left(^{*}\right)$ and **indicated $p<0.05$ and 0.001 , respectively

miR-3185 (Fig. 5c). We predicted that there was a binding site between miR-3185, ATG5 and ATG7 (Fig. 5d, e). Luciferase reporter gene assay confirmed the binding of miR-3185 with ATG5 and ATG7 (Fig. 5f, g). The expression of ATG5 and ATG7 was increased in HTR8 cells infected LV5-Inc02527 compared to that infected LV5-NC (Fig. 5h, i). The expression of ATG5 and ATG7 was decreased in HTR8 cells infected LV3-lnc02527-1 or LV3-Inc02527-2 compared to that infected LV3-NC (Fig. 5j, k). This data suggested that Linc02527 promoted autophagy by sponging miR-3185.

\section{Linc02527 directly binding to YBX1 and activated P21}

To further explore the mechanism of Linc02527 regulation autophagy. The RNA pull down assay was performed. Then using SDS-PAGE gel electrophoresis and Silver stain six differential bands were obtained(Fig. 6a). Using MALDI-TOF-MS, some protein including YBX1 were identified(S3). It indicated that YBX1 was directly bond to Linc02527(Fig. 6a-c).We confirmed that YBX1 was directly bond to Linc02527 through RNA pull down assay (Fig. 6f). The expression of YBX1 was increased in HTR8 cells infected LV5-Inc02527 compared to that infected LV5-NC(Fig. 6d). The expression of YBX1 was decreased in HTR8 cells infected LV3-lnc02527-1 or LV3-Inc02527-2 compared to that infected LV3-NC(Fig. 6e). Previous study reported that $\mathrm{P} 21$ pathway was activated by $\mathrm{YBX}^{26}$. P21 pathway can promotes autophagy ${ }^{27-29}$. P21 was elevated when over-expression of Linc02527. P21 was downregulated when silencing Linc02527 expression(Fig. 6d, e). So, we guessed Linc02527 bond to and stabilized YBX1, then activated P21 pathway and prompted autophagy.

\section{Autophagy was involved in growth of fetal mouse}

We have demonstrated Linc02527 promoted autophagy in vitro. However, we did not find homologous sequence of Linc02527 in rat and mouse. We observed autophagy was activated in ICP. So, we detected whether autophagy was activated in ICP in the rat model. The serum TBA, ALT and AST was increased in ICP in the rat model (Fig. 7a-c). The LC3-II, ATG5 and ATG7 expression was elevated in the placenta of ICP rat compared to normal group (Fig. 7d). This data revealed that autophagy was activated in ICP rat. We also observed YBX1 and P21 was elevated in the placenta of ICP rat compared to normal group (Fig. 7d). Then, we explore whether activated autophagy or inhibited autophagy can affect the growth of C57 mouse. We observed that the growth of C57 mouse was retarded when autophagy was activated using rapamycin compared to normal group (Fig. 7E1-2). Compared with the normal group, the growth of C57 mouse is not different when autophagy was inhibited using chloroquine (Fig. 7E3). The inhibition of growth by rapamycin is partly relieved through adding chloroquine (Fig. 7E4). The number of fetuses is no significant difference between normal control group, rapamycin group, chloroquine group and rapamycin + chloroquine group (Fig. 7f).The fetuses birth weight, body length and placenta weight were decreased in rapamycin group than that of normal control group (Fig. $7 \mathrm{~g}-\mathrm{i}$ ). The fetuses birth weight, body length and placenta weight were decreased in rapamycin group than that of normal control group (Fig. $7 \mathrm{~g}-\mathrm{i}$ ). The fetuses birth weight, body length and placenta weight were decreased in rapamycin + chloroquine group than that of rapamycin group (Fig. $7 \mathrm{~g}-\mathrm{i}$ ). There was not significant difference between chloroquine group and normal control group (Fig. $7 \mathrm{~g}-\mathrm{i}$ ). These data revealed that the growth of $\mathrm{C} 57$ mouse was repressed when autophagy was activated. In normal condition, inhibited autophagy using chloroquine did not affect the growth of C57 mouse. However, in the condition of autophagy was activated, inhibited autophagy using chloroquine can improve the growth of C57 mouse. Rapamycin caused fetal growth retardation, possibly due to inhibition of the mTOR pathway.

\section{Discussion}

In the present research, we found that Linc02527 was increased expression in serum and placenta of ICP patients. Linc02527 promoted autophagy in HTR8 cells. Linc02527 regulated ATG5 and ATG7 though sponging miR-3185. Linc02527 directly binding to YBX1 and activated P21, then the autophagy was promoted. We also found that regulation of autophagy was mediated the growth of fetal mouse. 


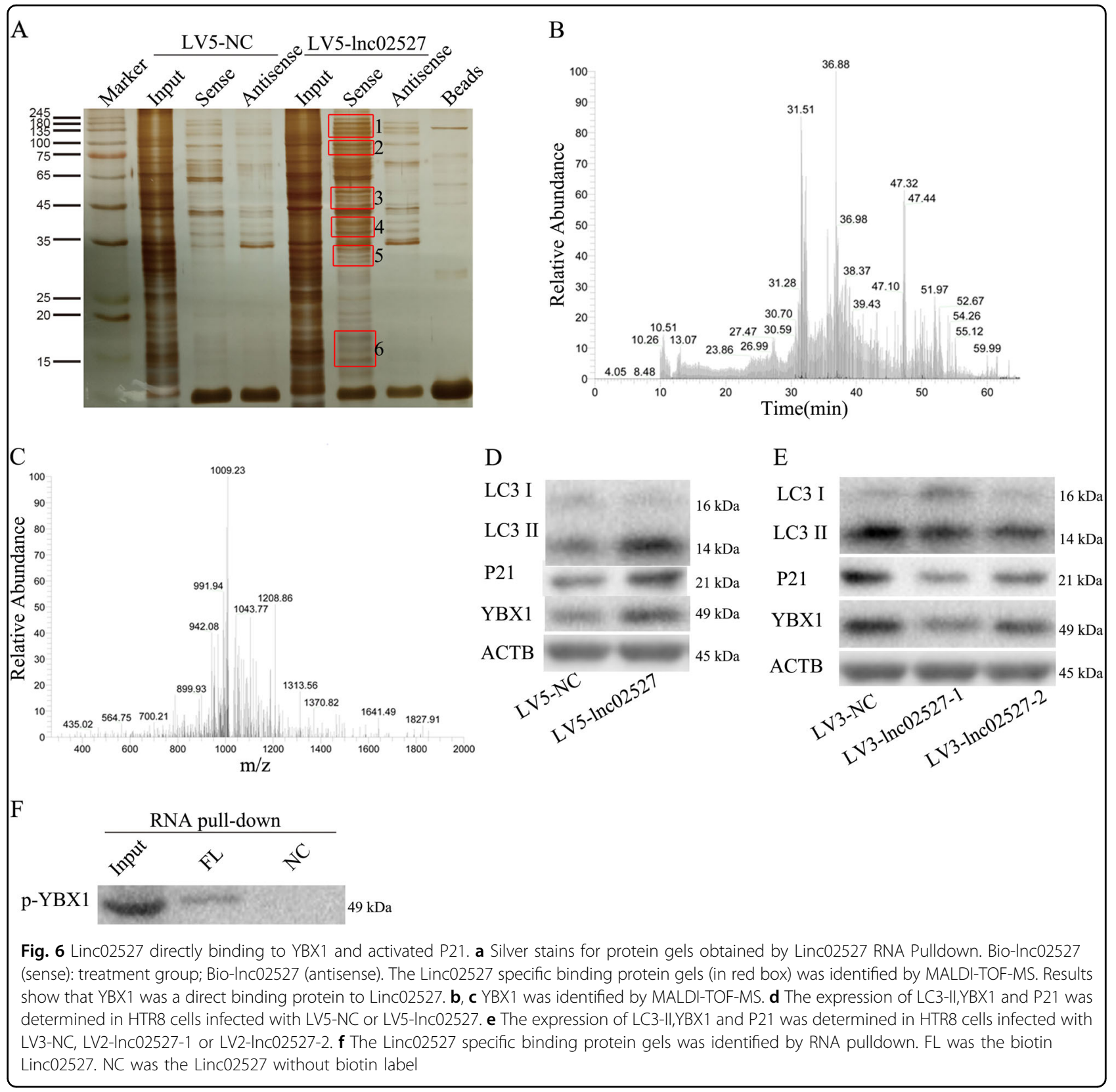

Linc02527 was located in 6q21. In 27 different human tissues, it is distinctive high-expression in placenta and testis ${ }^{30}$. In this study, we observed Linc02527 expression was increased in the placenta and serum of ICP patients. The serum TBA, CG, ALT and AST was elevated in ICP patients. The serum TBA and CG were diagnostic criteria of $\mathrm{ICP}^{31-33}$. The expression of Linc02527 was positively with the serum TBA, CG, ALT and AST. So, Linc02527 is a potential biomarker of Intrahepatic Cholestasis of Pregnancy(ICP).

In this research, we noted that Linc02527 was not involved in apoptosis. However, it promoted autophagy and proliferate. Many studies have demonstrated that
LncRNA regulated the target genes by sponging miRNAs ${ }^{34-36}$. ATG5 and ATG7 are two important moleculars to promote autophagy ${ }^{37-41}$. We found miR-3185 inhibited autophagy by targeting ATG5 and ATG7. Linc02527 directly bond to and down-regulated miR-3185. Our data revealed Linc02527 regulated ATG5 and ATG7 through sponging miR-3185. YBX1 regulates tumor growth via CDC25a pathway in human lung cancer ${ }^{42}$. IncRNA GAS5 enhances G1 cell cycle arrest via binding to YBX1 to regulate $\mathrm{p} 21$ expression in stomach cancer ${ }^{26}$. Phosphorylation of YBX1 activates NF- $\kappa B$ in colon cancer. We found Linc02527 directly bond to and stabilized YBX1. At the same time, Linc02527 increased the expression of P21. 


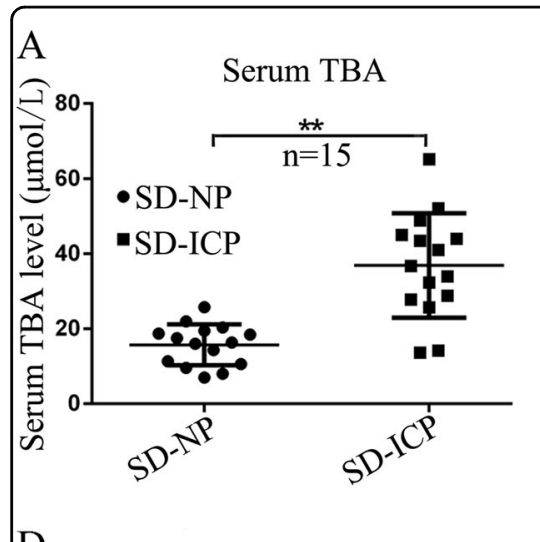

$\mathrm{D}$

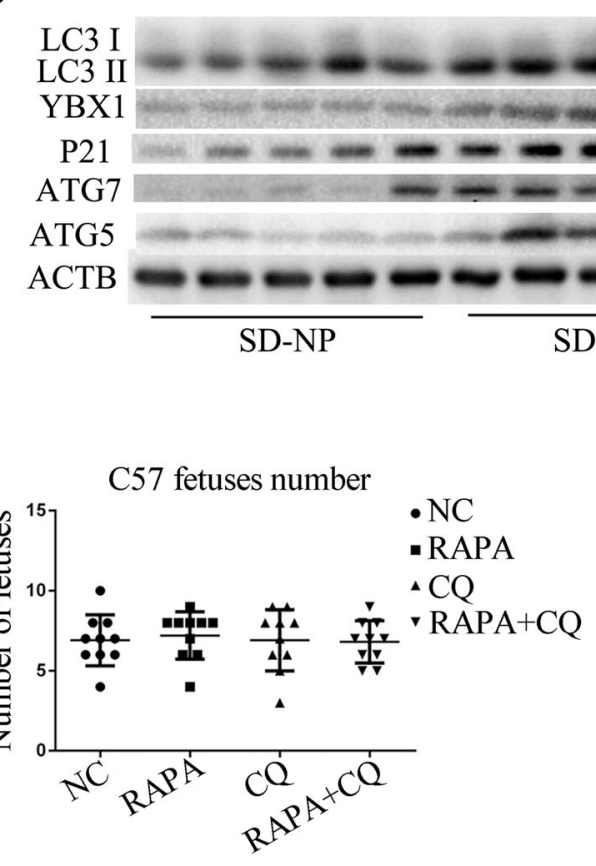

$\mathrm{H} \quad \mathrm{C} 57$ fetuses body length

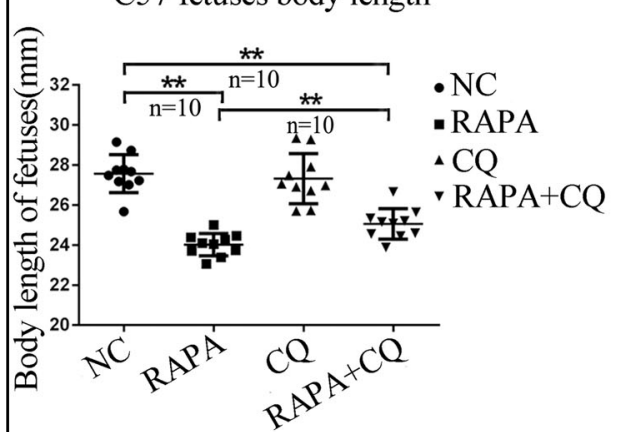

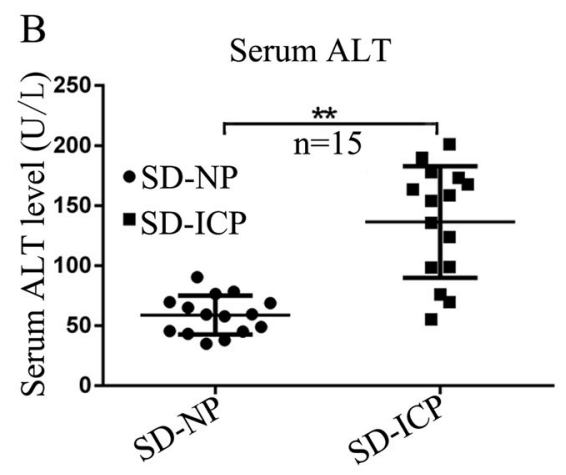

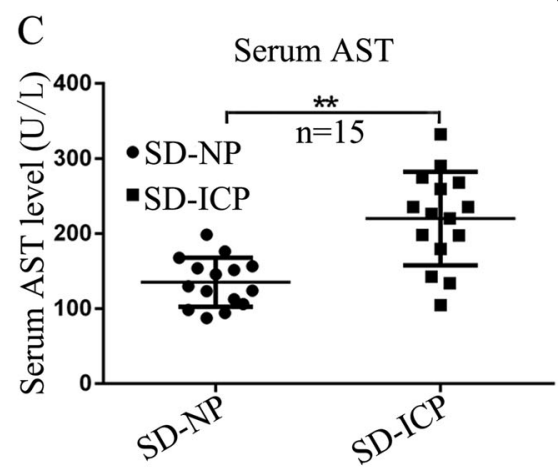

E

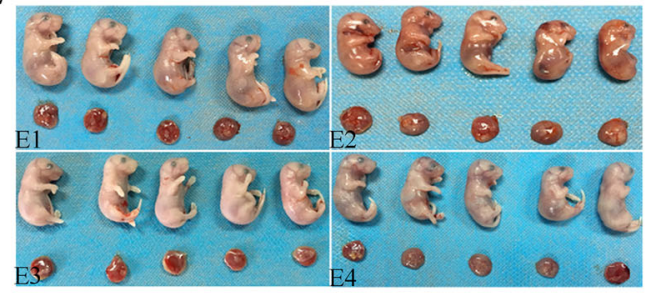

G C57 fetuses birthweight

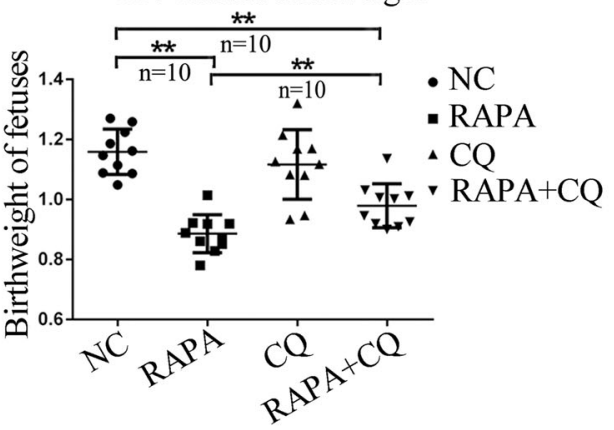

I $\quad$ 57 placenta weight

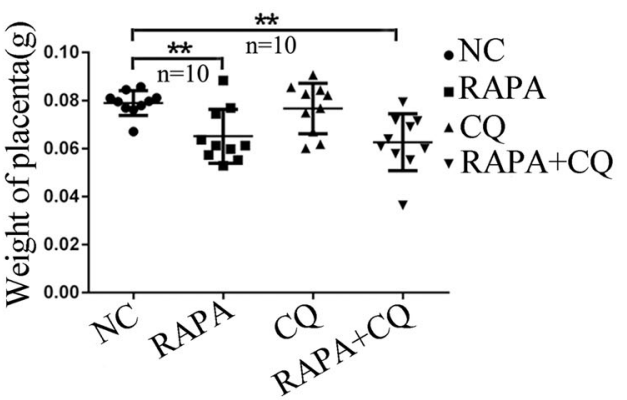

Fig. 7 Autophagy was involved in growth of fetal mouse. a-c The serum TBA, ALT and AST were detected in in ICP in the rat model. $\mathbf{d}$ The expression of LC3-II was detected in the placenta of ICP rat or NP group(normal control). e-i Forty C57 pregnant mice were randomly divided into four groups: group NC(E1), group RAPA(E2), group $C Q(E 3)$ and group $C Q+R A P A(E 4)$. From day 12 of gestation of gestation, mice in group NC were injected intraperitoneally with normal saline for 6 days consecutively. Group RAPA were injected intraperitoneally with rapamycin $(5 \mathrm{mg} / \mathrm{kg})$. Group CQ were injected intraperitoneally with chloroquine (50 mg/kg). Group RAPA + CQ were injected intraperitoneally with rapamycin $(5 \mathrm{mg} / \mathrm{kg})$ and chloroquine $(50 \mathrm{mg} / \mathrm{kg}$ ). On day 21 of gestation, the pregnant mice were sacrificed. Then, the fetal mice was calculated for body weight, fetus number, bodylenth and placenta weight. Error bars represented standard error. Asterisks $\left(^{*}\right)$ and ${ }^{* *}$ indicated $p<0.05$ and 0.001 , respectively 
P21 pathway can promotes autophagy ${ }^{27-29}$. So, we guessed Linc02527 bond to and stabilized YBX1, then activated P21 pathway and prompted autophagy. These results indicated that Linc02527 was a potential target for ICP.

Autophagy is an evolutionarily conserved catalytic process by which cytoplasmic components including damaged macromolecules and organelles are degraded ${ }^{43}$. An increased autophagosome formation was observed in placenta from preeclampsia and $\mathrm{FGR}^{44}$. In this study, we found LC3-II expression was elevated in placenta from ICP. The expression of LC3-II also was increased in the placenta of ICP rat. This result indicated that autophagy was activated in ICP. Autophagy has an important role in early embryo development. Increased embryonic or fetal death caused by inactivation of autophagy related genes in mouse. Atg5 deficient mice were fertilized normally, but manifested embryonic lethality at last ${ }^{45}$. Beclin1 deficient mice embryos also die as early as embryonic day $7.5^{46}$. Previous study has demonstrated chloroquine and hydroxychloroquine inhibit bladder cancer cell growth by targeting basal autophagy ${ }^{47}$. Hydroxychloroquine (HCQ) inhibits autophagy and sensitize some cancer cells to chemotherapy ${ }^{48}$. However, as an inhibitor of autophagy, HCQ reduces neonatal morbidity in women with SLE by significantly decreasing the rate of prematurity and intrauterine growth restriction ${ }^{49}$. We found chloroquine (CQ) did not increase the amount of fetal mice. But induced autophagy using rapamycin, the development of fetal mice was retard and the fetuses birth weight, body length and placenta weight were decreased. Chloroquine (CQ) can rescue the retarded development and increase the fetuses birth weight, body length and placenta weight at the condition of activated autophagy. Based on these results, we guessed that activated autophagy impaired the growth of fetal mice. Inhibited autophagy promoted the growth of fetus at the condition of activated autophagy. Chloroquine is a potential drug of ICP.

To our knowledge, this is the first time reported that autophagy was activated in ICP. The expression of Linc02527 was increased in placenta and serum in ICP patients. Linc02527 bond to and stabilized YBX1, then activated P21 pathway and prompted autophagy. We also found Chloroquine (CQ) can rescue the retarded development at the condition of activated autophagy. So, Linc02527 was a potential biomarker and target for ICP. Chloroquine may be a drug for treating ICP.

\section{Acknowledgements}

We thank members of our laboratory for technical help and stimulating discussions. This work was supported by the Natural Science Foundation of Chongqing (CSTC 2012JJB10030); the National Science Foundation of China (81172492); the National Science Foundation of China (81501220); and Chongqing Municipal Health and Family Planning Commission Medical Research Fund(2017MSXM031).
Conflict of interest

The authors declare that they have no conflict of interest.

\section{Publisher's note}

Springer Nature remains neutral with regard to jurisdictional claims in published maps and institutional affiliations.

Supplementary Information accompanies this paper at (https://doi.org/ 10.1038/s41419-018-1013-z).

Received: 25 May 2018 Revised: 28 August 2018 Accepted: 29 August 2018 Published online: 24 September 2018

\section{References}

1. Trauner, M., Fickert, P. \& Wagner, M. MDR3 (ABCB4) defects: a paradigm for the genetics of adult cholestatic syndromes. Semin. Liver Dis. 27, 77-98 (2007).

2. Allen, A. M. et al. The Epidemiology of Liver Diseases Unique to Pregnancy in a US Community: A Population-Based Study. Clin. Gastroenterol. Hepatol. 14, 287-294.e1 (2016). 2.

3. Ahmed, K. T., Almashhrawi, A. A., Rahman, R. N., Hammoud, G. M. \& Ibdah, J. A. Liver diseases in pregnancy: diseases unique to pregnancy. World J. Gastroenterol. 19, 7639-7646 (2013).

4. Almashhrawi, A. A., Ahmed, K. T., Rahman, R. N., Hammoud, G. M. \& Ibdah, J. A. Liver diseases in pregnancy: diseases not unique to pregnancy. World J. Gastroenterol. 19, 7630-7638 (2013).

5. Klionsky, D. J. Why do we need to regulate autophagy (and how can we do it)? A cartoon depiction. Autophagy. 14, 1661-1664 (2018).

6. Talukdar, S. et al. Regulation of protective autophagy in anoikis-resistant glioma stem cells by SDCBP/MDA-9/Syntenin. Autophagy. 14, 1845-1846 (2018).

7. Urano, Y. et al. 6-Hydroxydopamine induces secretion of PARK7/DJ-1 via autophagy-based unconventional secretory pathway. Autophagy. 16, 10-16 (2018) https://doi.org/10.1080/15548627.2018.1493043.

8. Kim, B. W. et al. The C-terminal region of ATG101 bridges ULK1 and Ptdlns3K complex in autophagy initiation. Autophagy. 16, 1-13 (2018) https:/doi.org/ 10.1080/15548627.2018.1504716.

9. Vantaggiato, C. et al. ZFYE26/SPASTIZIN and SPG11/SPATACSIN mutations in hereditary spastic paraplegia types AR-SPG15 and AR-SPG11 have different effects on autophagy and endocytosis. Autophagy. 16, 1-24 (2018) https:/doi. org/10.1080/15548627.2018.1507438

10. Zhou, X. et al. Impaired mitochondrial fusion, autophagy, biogenesis and dysregulated lipid metabolism is associated with preeclampsia. Exp. Cell Res. 359, 195-204 (2017).

11. Akaishi, R. et al. Autophagy in the placenta of women with hypertensive disorders in pregnancy. Placenta 35, 974-980 (2014).

12. Sun, Z., Wu, X. Y. \& Wu, C. L. The association between LncRNA HOTAIR and cancer lymph node metastasis and distant metastasis: a meta-analysis. Neoplasma 65, 178-184 (2018).

13. Zhao, Y. et al. The IncRNA MACC1-AS1 promotes gastric cancer cell metabolic plasticity via AMPK/Lin28 mediated mRNA stability of MACC1. Mol. Cancer 17, 69 (2018).

14. Fang, Z., Chen, W., Yuan, Z., Liu, X. \& Jiang, H. LncRNA-MALAT1 contributes to the cisplatin-resistance of lung cancer by upregulating MRP1 and MDR1 via STAT3 activation. Biomed. Pharmacother. 101, 536-542 (2018).

15. Han, D., Wang, J. \& Cheng, G. LncRNA NEAT1 enhances the radio-resistance of cervical cancer via miR-193b-3p/CCND1 axis. Oncotarget 9, 2395-2409 (2018).

16. He, X. et al. LncRNAs expression in preeclampsia placenta reveals the potential role of LnCRNAs contributing to preeclampsia pathogenesis. PLOS ONE 8 , e81437 (2013).

17. Penkala, I. et al. IncRHOXF1, a Long Noncoding RNA from the X Chromosome That Suppresses Viral Response Genes during Development of the Early Human Placenta. Mol. Cell. Biol. 36, 1764-1775 (2016).

18. Xu, W. et al. The Effect of and Mechanism Underlying Autophagy in Hepatocellular Carcinoma Induced by $\mathrm{CH} 12$, a Monoclonal Antibody Directed Against Epidermal Growth Factor Receptor Variant III. Cell. Physiol. Biochem. 46, 226-237 (2018)

19. Guo, Y., Shan, Q. Q., Gong, P. Y. \& Wang, S. C. The autophagy induced by curcumin via MEKJERK pathway plays an early anti-leukemia role in human 
Philadelphia chromosome-positive acute lymphoblastic leukemia SUP-B15 cells. J. Cancer Res Ther. 14, S125-125S131 (2018).

20. Wang, Y. et al. Metformin induces autophagy and GO/G1 phase cell cycle arrest in myeloma by targeting the AMPK/mTORC1 and mTORC2 pathways. J. Exp. Clin. Cancer Res. 37, 63 (2018).

21. Shen, L. et al. [Enhancing the ability of autophagy and proliferation of bone marrow mesenchymal stem cells by interleukin-8 through Akt-STAT3 pathway in hypoxic environment]. Sheng Wu Gong. Cheng Xue Bao 32, 1422-1432 (2016).

22. Ding, $\mathrm{C} . \mathrm{H}$. et al. The HNF1a-regulated IncRNA HNF1A-AS1 reverses the malignancy of hepatocellular carcinoma by enhancing the phosphatase activity of SHP-1. Mol. Cancer 17, 63 (2018).

23. Chen, J. F. et al. STAT3-induced IncRNA HAGLROS overexpression contributes to the malignant progression of gastric cancer cells via mTOR signal-mediated inhibition of autophagy. Mol. Cancer 17, 6 (2018).

24. Li, L. et al. Low expression of IncRNA-GAS5 is implicated in human primary varicose great saphenous veins. PLOS ONE 10, e0120550 (2015).

25. Boyer-Guittaut, M. et al. The role of GABARAPL1/GEC1 in autophagic flux and mitochondrial quality control in MDA-MB-436 breast cancer cells. Autophagy 10, 986-1003 (2014).

26. Liu, Y. et al. IncRNA GAS5 enhances $G 1$ cell cycle arrest via binding to YBX1 to regulate p21 expression in stomach cancer. Sci. Rep. 5, 10159 (2015).

27. Hong, S. \& Kim, M. M. IGFBP-3 plays an important role in senescence as an aging marker. Environ. Toxicol. Pharmacol. 59, 138-145 (2018).

28. Jung, D. et al. Quinacrine upregulates p21/p27 independent of p53 through autophagy-mediated downregulation of p62-Skp2 axis in ovarian cancer. Sci. Rep. 8, 2487 (2018).

29. Beyfuss, K. \& Hood, D. A. A systematic review of p53 regulation of oxidative stress in skeletal muscle. Redox Rep. 23, 100-117 (2018).

30. Fagerberg, L. et al. Analysis of the human tissue-specific expression by genome-wide integration of transcriptomics and antibody-based proteomics. Mol. Cell Proteom. 13, 397-406 (2014).

31. Chen, Z., Shen, Z., Hu, L., Lu, M. \& Feng, Y. Identification of Matrix Metalloproteinase-2 and 9 as Biomarker of Intrahepatic Cholestasis of Pregnancy. Ann. Hepatol. 16, 291-296 (2017).

32. Zhang, Y., Lu, L., Victor, D. W., Xin, Y. \& Xuan, S. Ursodeoxycholic Acid and Sadenosylmethionine for the Treatment of Intrahepatic Cholestasis of Pregnancy: A Meta-analysis. Hepat. Mon. 16, e38558 (2016).

33. Pařizek, A. et al. A Comprehensive Evaluation of Steroid Metabolism in Women with Intrahepatic Cholestasis of Pregnancy. PLoS ONE 11, e0159203 (2016).

34. Zhang, $K$. et al. LnCRNA FLVCR1-AS1 acts as miR-513c sponge to modulate cancer cell proliferation, migration, and invasion in hepatocellular carcinoma. J Cell Biochem. 119, 6045-6056 (2018).
35. Liang, L. et al. LnCRNA HCP5 promotes follicular thyroid carcinoma progression via miRNAs sponge. Cell Death Dis. 9, 372 (2018).

36. Zhang, Z. K. et al. A newly identified IncRNA MAR1 acts as a miR-487b sponge to promote skeletal muscle differentiation and regeneration. J Cachexia Sarcopenia Muscle. 9, 613-626 (2018).

37. Zhang, $\mathrm{K}$. et al. PU.1/microRNA-142-3p targets ATG5/ATG16L1 to inactivate autophagy and sensitize hepatocellular carcinoma cells to sorafenib. Cell Death Dis. 9, 312 (2018).

38. Wang, N. et al. MicroRNA-9a-5p Alleviates Ischemia Injury After Focal Cerebral Ischemia of the Rat by Targeting ATG5-Mediated Autophagy. Cell. Physiol. Biochem. 45, 78-87 (2018).

39. Hu, Z. Y., Chen, B., Zhang, J. P. \& Ma, Y. Y. Up-regulation of autophagy-related gene 5 (ATG5) protects dopaminergic neurons in a zebrafish model of Parkinson's disease. J. Biol. Chem. 292, 18062-18074 (2017).

40. Qiang, L. et al. Autophagy gene ATG7 regulates ultraviolet radiationinduced inflammation and skin tumorigenesis. Autophagy 13, 2086-2103 (2017).

41. Su, L. Y. et al. Atg5- and Atg7-dependent autophagy in dopaminergic neurons regulates cellular and behavioral responses to morphine. Autophagy 13, 1496-1511 (2017).

42. Zhao, S. et al. YBX1 regulates tumor growth via CDC25a pathway in human lung adenocarcinoma. Oncotarget 7, 82139-82157 (2016).

43. $\mathrm{Hu}$, J. et al. MARCH5 RNA promotes autophagy, migration, and invasion of ovarian cancer cells. Autophagy. 13, 333-344 (2017).

44. Oh, S. Y. \& Roh, C. R. Autophagy in the placenta. Obstet. Gynecol. Sci. 60, 241-259 (2017)

45. Tsukamoto, S. et al. Autophagy is essential for preimplantation development of mouse embryos. Science 321, 117-120 (2008).

46. Yue, Z., Jin, S., Yang, C., Levine, A. J. \& Heintz, N. Beclin 1, an autophagy gene essential for early embryonic development, is a haploinsufficient tumor suppressor. Proc. Natl Acad. Sci. USA 100, 15077-15082 (2003).

47. Lin, Y. C. et al. Chloroquine and hydroxychloroquine inhibit bladder cancer cell growth by targeting basal autophagy and enhancing apoptosis. Kaohsiung J. Med. Sci. 33, 215-223 (2017).

48. Wang, Y. et al. Significantly enhanced tumor cellular and lysosomal hydroxychloroquine delivery by smart liposomes for optimal autophagy inhibition and improved antitumor efficiency with liposomal doxorubicin. Autophagy 12, 949-962 (2016).

49. Leroux, M. et al. Impact of hydroxychloroquine on preterm delivery and intrauterine growth restriction in pregnant women with systemic lupus erythematosus: a descriptive cohort study. Lupus 24, 1384-1391 (2015). 